\title{
Effects of Granulocyte Colony-Stimulating Factor on Patients with Liver Failure: a Meta-Analysis
}

\author{
Qiao Yang*1, Ying Yang ${ }^{2}$, Yu Shi ${ }^{2}$, Fangfang Lv ${ }^{1}$, Jiliang $\mathrm{He}^{3}$ and Zhi Chen ${ }^{2}$ \\ ${ }^{1}$ Department of Infectious Diseases, Sir Run Run Shaw Hospital, School of Medicine, Zhejiang University, Hangzhou, Zhejiang, \\ China; ${ }^{2}$ State Key Lab of Diagnosis and Treatment of Infectious Diseases, Collaborative Innovation Center for Diagnosis and \\ Treatment of Infectious Disease, First Affiliated Hospital, Zhejiang University School of Medicine, Hangzhou, Zhejiang, China; \\ ${ }^{3}$ Institute of Environmental Medicine, Medical College, Zhejiang University, Hangzhou, Zhejiang, China
}

\section{Abstract}

Background and Aims: It remains controversial whether granulocyte colony-stimulating factor (G-CSF) prolongs survival in liver failure (LF) patients. This meta-analysis was performed to evaluate the effect of G-CSF on patients with LF. Methods: PubMed, EMBASE, and Web of Science databases were searched to identify English language randomized controlled trials comparing G-CSF with control therapy published before14 February 2015. A meta-analysis was performed to examine changes in liver function and patient survival. The association was tested using odds ratio (OR) or risk ratio (RR) with $95 \%$ confidence intervals (CI). Results: Five randomized controlled trials were eligible for the meta-analysis. Significant amelioration of prothrombin time and total bilirubin in LF patients was attributed to G-CSF therapy (OR, $-0.064 ; 95 \% \mathrm{CI},-0.481$ to $0.353 ; p<0.001$; and $\mathrm{OR}$ $-0.803 ; 95 \% \mathrm{CI},-1.177$ to $-0.430 ; p=0.000$, respectively). Treatment with G-CSF resulted in improved Model for EndStage Liver Disease and Child-Turcotte-Pugh scores (OR, $-1.741 ; 95 \% \mathrm{CI},-2.234$ to $-1.250 ; p=0.000$; and $\mathrm{OR}_{\text {, }}$ $-0.830,95 \% \mathrm{CI},-1.194$ to $-0.465 ; p=0.000$, respectively). A lower incidence of sepsis was found in patients treated with $\mathrm{G}$ CSF (RR, $0.367 ; 95 \%$ CI, 0.158 to $0.854 ; p=0.020$ ). G-CSF therapy significantly increased survival rate in LF patients $(R R$, $2.25 ; 95 \% \mathrm{CI}, 1.517$ to $3.338 ; p=0.000)$. Conclusions: The results of this meta-analysis indicate that G-CSF treatment in patients with LF significantly improved liver function, reduced the incidence of sepsis, and prolonged short-term survival. (C) 2016 The Second Affiliated Hospital of Chongqing Medical University. Published by XIA \& HE Publishing Inc. All rights reserved.

\section{Introduction}

Liver failure (LF) is a syndrome characterized by severe hepatic decompensation, resulting in jaundice, infection, hepatic encephalopathy, hepatorenal syndrome, and multisystem organ failure. ${ }^{1}$ The costs associated with intensive care and short-term mortality in LF patients have remained quite high over the past few decades. ${ }^{2}$ There is, therefore, an urgent need for more effective adjuvant therapies against LF.

Granulocyte colony-stimulating factor (G-CSF) is a growth factor that stimulates bone marrow to produce granulocytes and stem cells and release them into the circulation. ${ }^{3} \mathrm{~A}$ significant body of observational and randomized data have indicated a potential role of G-CSF for end-stage liver diseases. ${ }^{4-7}$ Nevertheless, it remains uncertain whether G-CSF use can influence the prognosis for established patients with LF. Recently, several studies have shown that G-CSF mobilized $\mathrm{CD} 34^{+}$hematopoietic stem cells and improved liver regeneration in LF patients. ${ }^{8-12}$ Although the optimal time, dose, and duration of G-CSF treatment and which type of patients with LF are most likely to benefit from G-CSF treatment are unclear, data suggest that G-CSF may be a promising adjuvant therapy for patients with LF. In the present meta-analysis, the benefit of G-CSF use for patients with LF was assessed, providing evidence for the therapeutic use of G-CSF in patients with LF.

\section{Methods}

\section{Search strategy and selection of the papers}

Systematic searches of PubMed, Embase, and Cochrane databases for articles published before February 2015 were performed for possible publications. Reports cited in the references and relevant reviews were also searched, which included potentially missed studies. The following terms were used in the search procedure: ('liver failure' or 'hepatic failure' or 'fulminant hepatitis' or 'severe hepatitis' or 'end-stage liver disease') and ('Granulocyte colony-stimulating factor' or 'G-CSF'). The retrieved studies were carefully examined to exclude potential duplicates or overlapping data. Titles and abstracts selected from the initial search were first scanned, and the full papers of potential eligible studies were reviewed.

Eligibility of the studies was assessed independently by two investigators. The inclusion criteria of studies were the following: the outcomes of interest were liver function, clinical severity indices, the survival of liver or hepatic failure, and fulminant hepatitis or end-stage liver disease; the study of 
Qiao Y. et al: G-CSF therapy for liver failure

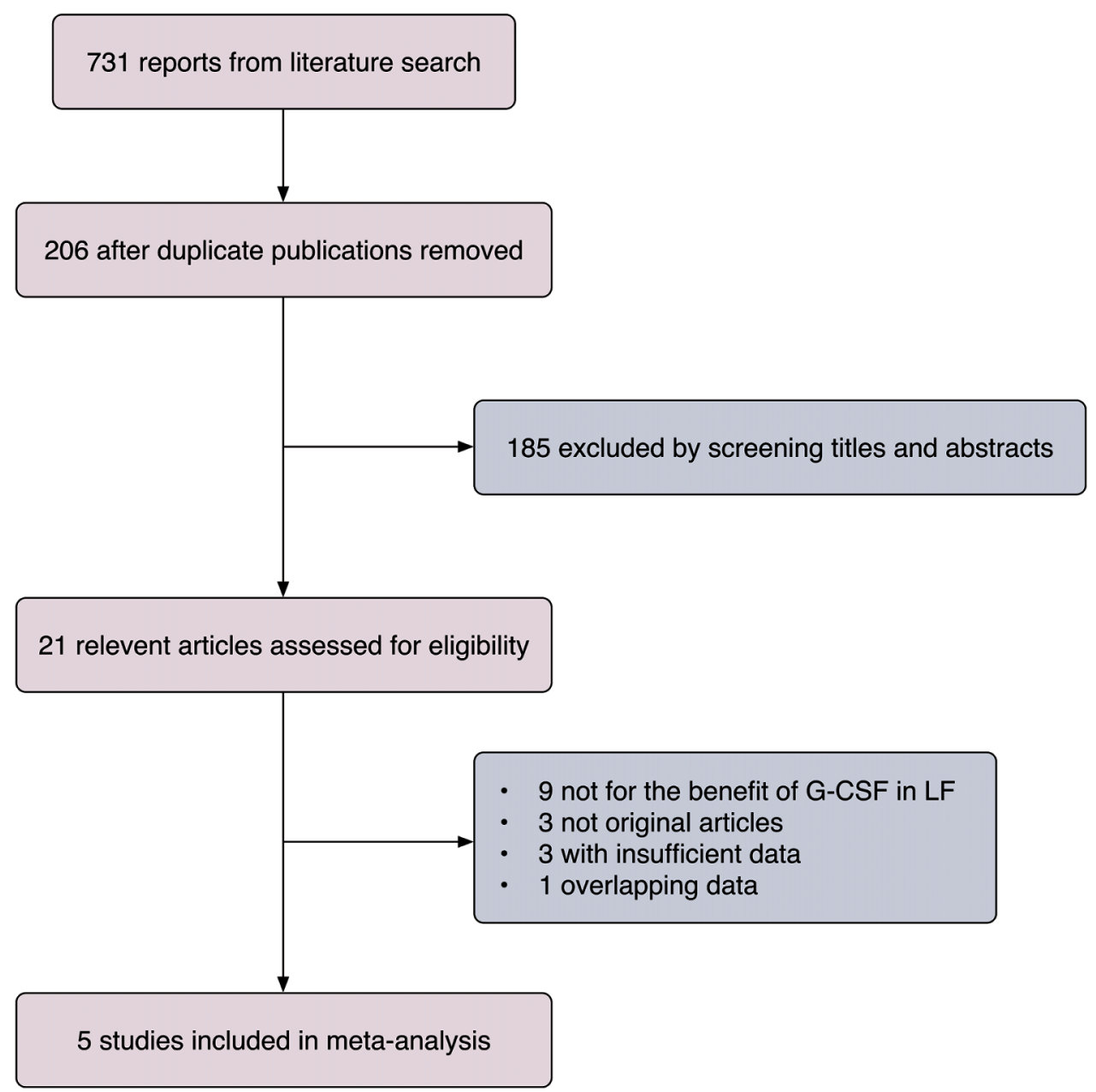

Fig. 1. Flow diagram of the selection process. Five studies assessed G-CSF use and outcomes in LF. Abbreviations: G-CSF, granulocyte colony-stimulating factor; LF, liver failure.

interest was G-CSF usage, with information available on the odds ratio (OR) or risk ratio (RR) estimates with $95 \%$ confidence intervals (CIs). Only studies published in English were included.

\section{Data extraction and quality assessment}

Data were extracted independently by two authors, and any discrepancies were resolved by discussion among the authors. The following information was obtained from each trial: publication details (title, the first author, origin of the study), study design, participants' details (the numbers of patients enrolled, participant demographics), the intervention details, the duration of follow-up, and primary outcomes (liver function, clinical severity indices, survival, and side effects).

Quality assessment of the included studies was conducted by two authors using the Jadad quality scale. ${ }^{13}$ Each study was evaluated by examining the allocation sequence generation, allocation concealment, blinding of outcome assessors, and reporting of patient withdrawal and dropout. Studies with scores more than 4 were defined as high-quality.

\section{Statistical methods analysis}

ORs were calculated to assess the advantage of G-CSF compared to control therapy. Rate ratios were calculated to show how many times higher the probability of death or the incidence of sepsis was in patients receiving G-CSF compared to those receiving controls therapies. RR with $95 \%$ CIs was calculated using a fixed- or random-effects model. Heterogeneity between individual studies was assessed by $I^{2}$ test, where a value greater than $50 \%$ was indicative of substantial heterogeneity. A random-effects model was used when the heterogeneity was significant, and a fixed-effects model was applied otherwise. Because of the limitations of test power when less than 10 studies are included, further statistical tests were not conducted. A $p$ value $<0.05$ was considered statistically significant. STATA 11.0 software was used in all analyses (Stat Corporation, College Station, Texas, USA). 


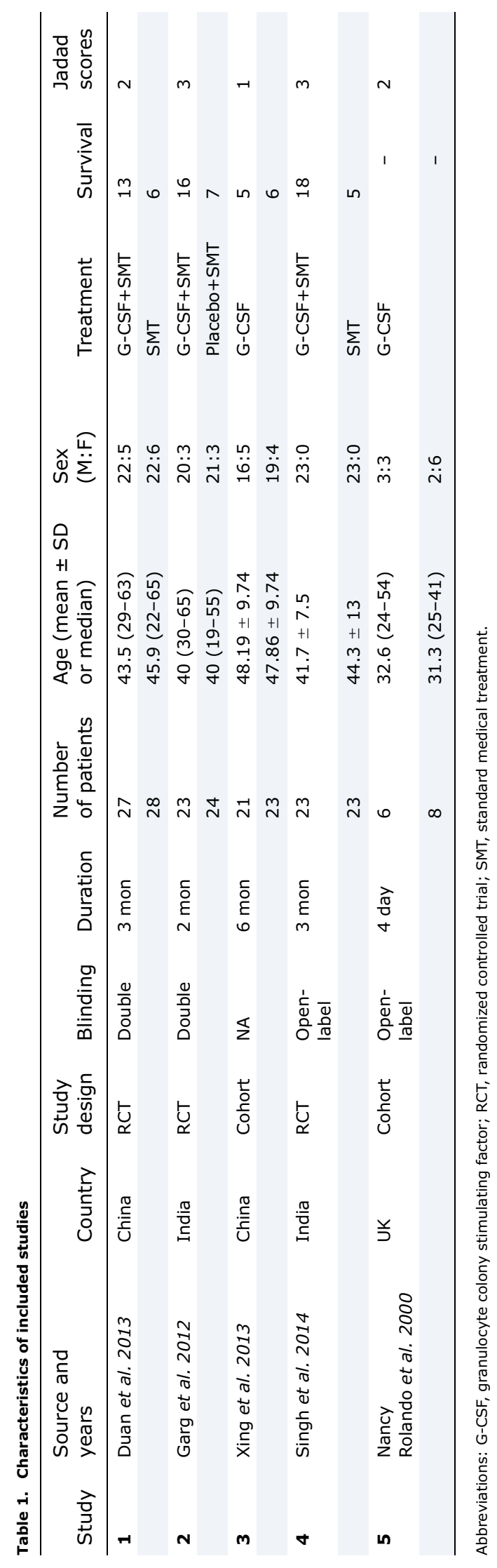


Qiao Y. et al: G-CSF therapy for liver failure

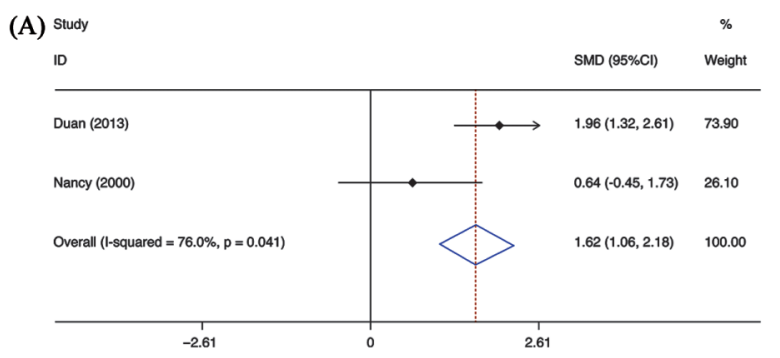

(B) Study

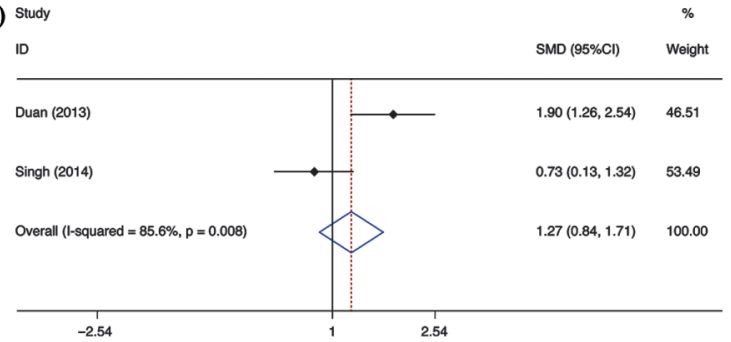

Fig. 2. Meta-analysis of the neutrophils and $\mathrm{CD}_{3} 4^{+}$cell counts in peripheral blood between G-CSF and control therapy. (A) Neutrophil counts in peripheral blood. (B) $\mathrm{CD}_{3}{ }^{+}$cell counts in peripheral blood. Abbreviation: G-CSF, granulocyte colony-stimulating factor.

\section{Results}

\section{Description of the included studies}

Our systematic literature search identified 731 articles for eligibility, of which 21 were potentially relevant reports after reviewing titles and abstracts. Among the remaining 21 fulltext articles, nine articles were excluded because the data did not involve the potential benefit of G-CSF in LF patients, three articles were excluded because they were not original articles, three articles were excluded because of an absence of acquired data, and one article was excluded because the same authors published overlapping data on the same patients. For those patients, clinical data was extracted by integrating the overlapping articles. In total, five articles met the inclusion criteria for the final meta-analysis (Fig. 1). ${ }^{8-12}$

Five clinical trials involving the potential effects of G-CSF in LF patients met the inclusion criteria, and the characteristics of these studies are shown in Table 1. Among these trials, two were conducted in India, ${ }^{9,11}$ two were conducted in China, 8,10 and one was conducted in the UK. ${ }^{12}$ Three of the studies were randomized controlled trials (RCTs), $, 9,9,11$ and two were prospective cohort studies. ${ }^{10,12}$ One study only enrolled patients with severe alcoholic hepatitis, ${ }^{11}$ one study admitted patients with LF due to acetaminophen overdose, ${ }^{12}$ two studies included only patients with hepatitis B virus (HBV)-associated $\mathrm{LF}^{8,10}$ and the remaining study included LF patients with various etiologies. ${ }^{9}$

\section{Mobilization of neutrophils and $\mathrm{CD}_{34}{ }^{+}$cells in peripheral blood}

Two of the studies assessed neutrophil counts after G-CSF administration. ${ }^{8,12}$ In one study, ${ }^{8}$ patients in the experimental groups were administered G-CSF subcutaneously at the dosage of $5 \mu \mathrm{g} / \mathrm{kg}$ per day for 6 consecutive days; peripheral neutrophil counts were quantified twice a week for 2 weeks. The data were extracted at the peak level. In the other study, ${ }^{12}$ patients were assigned to receive G-CSF at consecutive doses, and data on a safe and effective dose at $96 \mathrm{~h}$ were extracted. The pooled analysis represented an OR of 1.619 (95\% CI 1.062 to $2.175, p=0.000)$ with significant heterogeneity $\left(\mathrm{I}^{2}=76 \%, p=0.041\right)$ (Fig. 2A). Three studies ${ }^{8,9,11}$ evaluated $\mathrm{CD} 34^{+}$cells in peripheral blood; Garg et al. ${ }^{9}$ was excluded, as the required data were unavailable. The estimated pooled OR value showed that $\mathrm{CD} 34^{+}$cells were significantly increased in the peripheral blood of patients receiving G-CSF therapy ( OR $=1.273,95 \% \mathrm{CI}$ 0.836 to $1.710, p=0.000)$, with significant heterogeneity between the studies $\left(\mathrm{I}^{2}=85.6 \%, p=0.008\right)$ (Fig. $2 \mathrm{~B}$ ).

\section{Amelioration in liver function parameters}

Data on liver function parameters were available in three trials comparing G-CSF with control therapy. ${ }^{10,11}$ No significant difference was found in prothrombin time (PT) between G-CSF and control therapy groups (OR $=-0.064,95 \%$ $\mathrm{CI}-0.481$ to $0.353, p=0.764)$. No substantial heterogeneity was detected $\left(\mathrm{I}^{2}=61.4 \%, p=0.107\right)$ (Fig. $\left.3 \mathrm{~A}\right)$. For serum levels of total bilirubin (TBIL), ${ }^{9-11}$ the pooled OR value was significantly reduced in patients receiving $\mathrm{G}-\mathrm{CSF}(\mathrm{OR}=$ $-0.803,95 \%$ CI -1.177 to $-0.430, p=0.000)$, with significant heterogeneity across studies $\left(\mathrm{I}^{2}=94.0 \%, p=0.000\right)$ (Fig. 3B).

\section{Improvement in clinical severity indices}

The scores of Model for End-Stage Liver Disease (MELD) in LF patients for meta-analysis were available from two publications. ${ }^{8,9}$ MELD scores were significantly improved in G-CSF
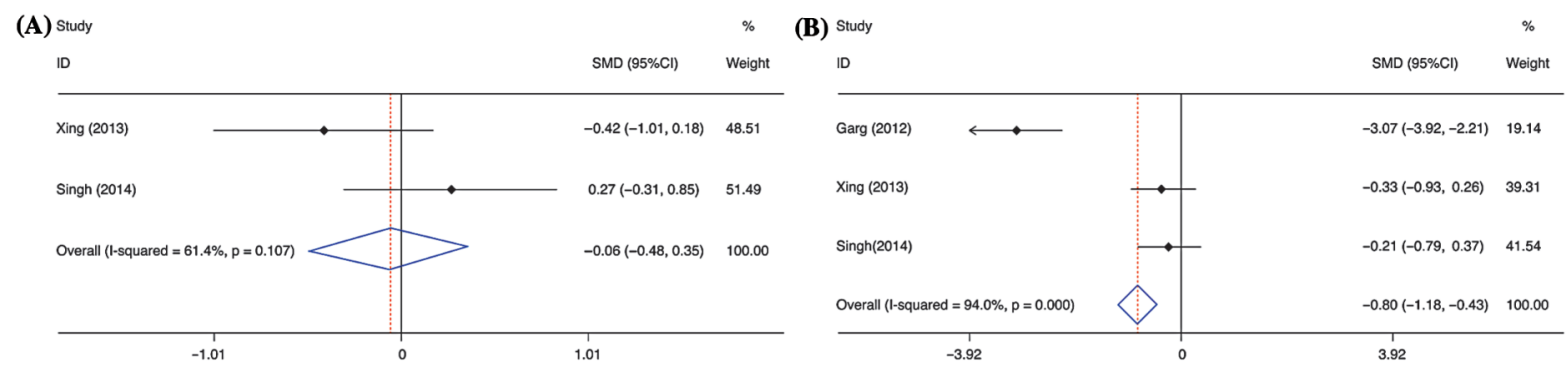

Fig. 3. Meta-analysis of the levels of prothrombin time and total bilirubin between G-CSF and control therapy. (A) The levels of prothrombin time. (B) The level of total bilirubin. Abbreviation: G-CSF, granulocyte colony-stimulating factor. 
Qiao Y. et al: G-CSF therapy for liver failure
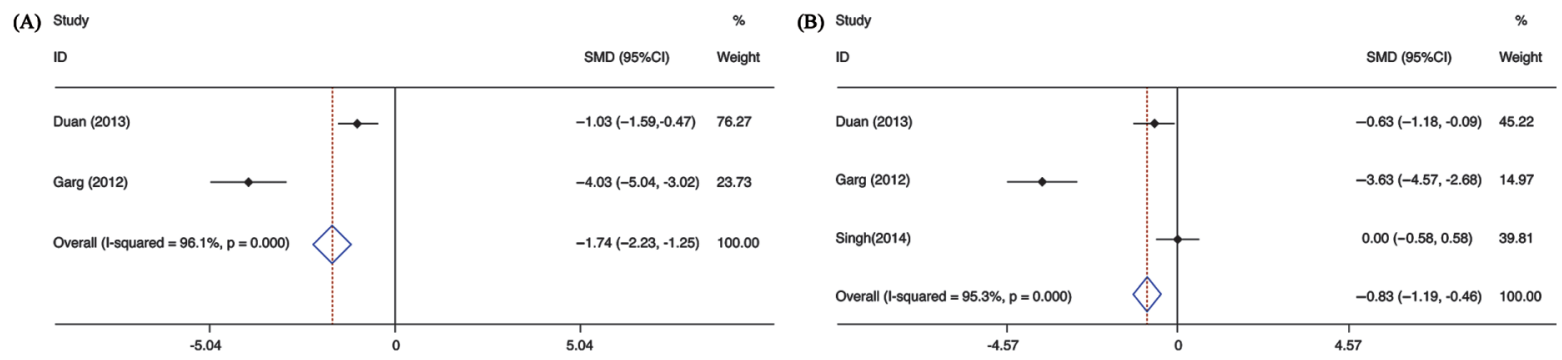

Fig. 4. Meta-analysis of MELD and CTP scores between G-CSF and control therapy. Abbreviations: CTP, Child-Turcotte-Pugh; MELD, Model for End-Stage Liver Disease; G-CSF, granulocyte colony-stimulating factor.

groups compared to control groups (pooled $\mathrm{OR}=-1.741$, $95 \% \mathrm{CI}-2.234$ to $-1.250, p=0.000)$, with significant heterogeneity between studies $\left(\mathrm{I}^{2}=96.1 \%, p=0.000\right)$ (Fig. 4A). The results of Child-Turcotte-Pugh (CTP) scores for the metaanalysis were obtained from three trials. ${ }^{8,9,11}$ The estimated pooled OR value showed that CTP scores were significantly decreased in G-CSF groups compared to the control groups (pooled OR $=-0.830,95 \% \mathrm{CI}-1.194$ to $-0.465, p=0.000$ ) (Fig. 4B). A significant heterogeneity was found among these studies $\left(\mathrm{I}^{2}=95.3 \%, p=0.000\right)$.

\section{Reduction in the occurrence of sepsis}

The results of analyses for the association between G-CSF use and the occurrence of sepsis are shown in Figure 5.8,9 The results demonstrated that the incidence of sepsis was lower in patients treated with G-CSF than with standard medical treatment (SMT) $(\mathrm{RR}=0.367,95 \% \mathrm{CI} 0.158$ to $0.854, p=0.020)$. No significant heterogeneity was found among the studies $\left(\mathrm{I}^{2}=0.0 \%, p=0.686\right)$.

\section{Prolongation in survival}

In four studies, the association between short-term survival benefit and G-CSF use in LF patients with various etiology was assessed. ${ }^{8-11}$ In total, 42 of $94 \mathrm{G}$-CSF users died from multiorgan failure, whereas 74 of 98 G-CSF non-users died. The pooled analysis of the survival benefit represented an RR of 2.25 ( $95 \%$ CI 1.517 to $3.338, p=0.000$ ), with no substantial heterogeneity across studies $\left(\mathrm{I}^{2}=30.1 \%, p=0.232\right)$. After excluding the study that applied case-control analysis of a cohort study, ${ }^{10}$ the RR increased even further for patients with G-CSF therapy (RR $=2.682,95 \%$ CI 1.732 to $4.151, p=0.000)$, indicating no heterogeneity among studies $\left(\mathrm{I}^{2}=0.0 \%, p=0.666\right)$ (Fig.6).

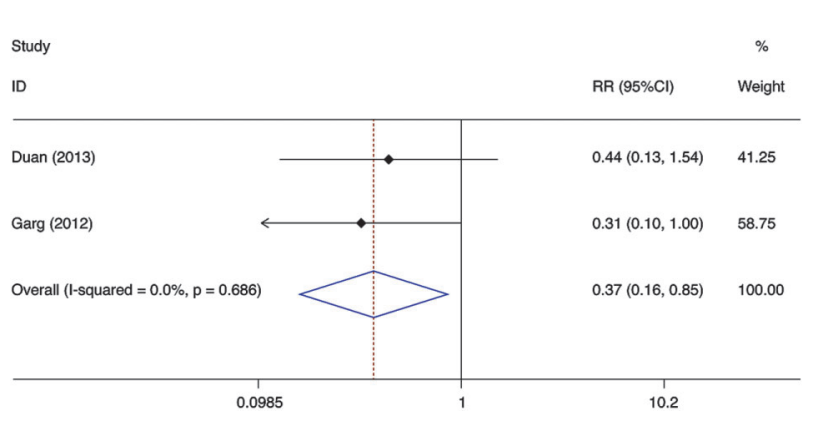

Fig. 5. Meta-analysis of the occurrence of sepsis between G-CSF and control therapy.

\section{Safety of G-CSF in LF patients}

No significant adverse reactions were reported in these studies. One patient had a high fever, and that dose of G-CSF was omitted and did not disturb the therapy. ${ }^{9}$

\section{Publication bias}

There was no evidence of publication bias in this study. However, because of the limited number of included studies, it is difficult to confirm whether publication bias existed in the current meta-analysis.

\section{Discussion}

This meta-analysis summarized the results of five studies on the clinical benefit of G-CSF in patients with LF. The results indicated that use of G-CSF improves liver function, alleviates clinical severity, and increases survival of LF due to a variety of causes.

LF is a life-threatening medical syndrome with high shortterm mortality. Liver transplantation remains the most effective therapy for the disease, but the shortage of donor organs limits its availability in most patients with LF; ${ }^{14}$ hence, it is imperative to develop novel therapeutic strategies for LF.

Promising results have been reported with G-CSF therapy in several high-quality clinical trials involving patients with various liver diseases. ${ }^{15-22}$ G-CSF mobilizes bone marrow hematopoietic stem cells to aid liver regeneration, and it is

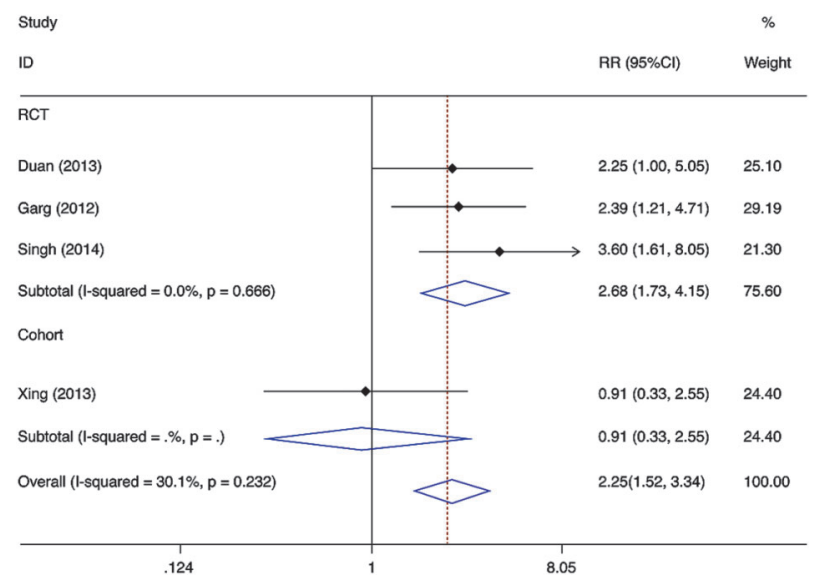

Fig. 6. Meta-analysis of the association between G-CSF use and liver failure mortality. 
most likely via this mechanism that G-CSF improves survival in acute and chronic LF. ${ }^{23-24}$ As suggested by previous studies, bone marrow-derived stems cells, together with hepatocytes and intrahepatic stem cells, may represent the third proliferative component of the liver regenerative process. ${ }^{5}$ In our review, we provided evidence that peripheral $\mathrm{CD} 34^{+}$cells in G-CSF groups were elevated significantly, which may also contribute to liver regeneration. In addition, G-CSF exerts autocrine and paracrine effects to promote the oval cell reaction in the liver, cooperatively improving the liver function. ${ }^{25}$

In addition, G-CSF modulates innate and adaptive immune response in LF patients. Several studies in vitro and in vivo have documented that G-CSF improved neutrophil function and decreased episodes of sepsis and sepsis-related death in LF patients. $8,9,21$ Our analysis also showed that peripheral neutrophil counts were higher and the incidence of sepsis was lower in G-CSF treated groups. Importantly, G-CSF therapy increased the frequencies of dendritic cells and reduced IFN- $\gamma$ secreting $C D 8^{+} T$ cells, thus reducing immune-mediated liver injury. ${ }^{26}$

Furthermore, we comprehensively analyzed in this metaanalysis the clinical severity indices of LF, and the results suggested that G-CSF therapy may result in progressive improvement in MELD and CTP scores at 2 to 3 months. In addition, patients with G-CSF therapy achieved significantly lower short term mortality compared with standard medical treatment only.

However, there were some confusing matters left unresolved, including the types of LF patients who responded well to G-CSF therapy and the optimal dose and duration of G-CSF adjuvant therapy. Meanwhile, attention should be given to the safety of G-CSF administration to induce bone marrow derived cell mobilization in patients with LF. Although G-CSF therapy was shown to be well tolerated in these studies, it is important to identify the optimal dose and duration of G-CSF that would achieve desirable hepatoprotective effects and the fewest adverse effects. Most of the included studies adopted the dosage of $5 \mu \mathrm{g} / \mathrm{kg} \cdot \mathrm{d}$ for 5 consecutive days. One of the studies included in this meta-analysis investigated the dose-response effect of G-CSF on patients with ALF due to acetaminophen overdose ${ }^{12}$ and found that G-CSF at the dose of $50 \mu \mathrm{g} / \mathrm{m}^{2}$ led to augmented neutrophil counts, which was similar to those achieved at higher doses, and fewer side effects as compared to higher doses of therapy. Because of the limited data, dose-response analysis for the association between G-CSF use and LF survival could not be conducted and the optimal duration of G-CSF in LF is still unclear.

The present meta-analysis is restricted by several limitations. First, the number of studies involved in the metaanalysis was small, and thus, the reliability of some of the subgroup analyses might be less than if the number of studies was greater. Second, the etiology of the underlying LF did not achieve homogeneity. Most of the etiologies in the analysis were alcohol, hepatitis $B$, and acetaminophen overdose. Due to the inadequate number of available studies, the subgroup analyses based on different etiology were not conducted. Thirdly, the dose and duration of G-CSF usage differed among different studies, and some data were not available from each study, which may cause less accurate estimates of the benefit of G-CSF therapy. Another drawback of the meta-analysis is the regional restriction of the included studies. The studies were conducted in Asia and Europe (two studies belonged to the mainland of China, two belonged to India, and one belonged to UK) and, therefore, the conclusion may not be generalized to other population.

In conclusion, the meta-analysis comprehensively analyzed the data on the benefit of G-CSF therapy for patients with LF, and the results suggested that G-CSF use in collaboration with SMT may improve LF survival. These findings have considerable importance in clinical practice since the mortality of LF is high and the availability of liver transplantation is not widely attainable. Future studies, especially large size, controlled longitudinal follow-up studies are necessary to strengthen the evidence supporting the use of G-CSF as an adjuvant therapy for LF survival.

\section{Acknowledgments}

This study was supported by the National Natural Science Foundation of China (81400622), the 12-5 State S\&T Projects of China (2012ZX10002007) and the Public Technology Research and social development project of Zhejiang Province China (2014C33177).

\section{Conflict of interest}

None

\section{Author contributions}

Conceived and designed the meta-analysis ( $\mathrm{QY}, \mathrm{FL}, \mathrm{ZC}$ ), analyzed the data (QY, $Y S, Y Y$ ), wrote the manuscript $(\mathrm{QY}, \mathrm{YY})$, revised the manuscript $(\mathrm{QY}, \mathrm{JH})$, checked the manuscript ( $F L, Z C)$.

\section{References}

[1] Jalan R, Gines P, Olson JC, Mookerjee RP, Moreau R, Garcia-Tsao G, et al. Acute-on chronic liver failure. J Hepatol 2012;57:1336-1348. doi: 10. 1016/j.jhep.2012.06.026.

[2] Olson JC, Wendon JA, Kramer DJ, Arroyo V, Jalan R, Garcia-Tsao G, et al. Intensive care of the patient with cirrhosis. Hepatology 2011;54:18641872. doi: 10.1002/hep.24622.

[3] Gianni AM, Siena S, Bregni M, Tarella C, Stern AC, Pileri A, et al. Granulocytemacrophage colony-stimulating factor to harvest circulating haemopoietic stem cells for autotransplantation. Lancet 1989;2:580-585. doi: 10.1016/ S0140-6736(89)90711-3.

[4] Theocharis SE, Papadimitriou LJ, Retsou ZP, Margeli AP, Ninos SS, Papadimitriou JD. Granulocyte-colony stimulating factor administration ameliorates liver regeneration in animal model of fulminant hepatic failure and encephalopathy. Dig Dis Sci 2003;48:1797-1803. doi: 10.1023/A:1025463532521.

[5] Gaia S, Smedile A, Omedè P, Olivero A, Sanavio F, Balzola F, et al. Feasibility and safety of G-CSF administration to induce bone marrow-derived cells mobilization in patients with end stage liver disease. J Hepatol 2006;45: 13-19. doi: 10.1016/j.jhep.2006.02.018.

[6] Spahr L, Lambert JF, Rubbia-Brandt L, Chalandon Y, Frossard JL, Giostra E, et al. Granulocyte-colony stimulating factor induces proliferation of hepatic progenitors in alcoholic steatohepatitis: a randomized trial. Hepatology 2008;48:221-229. doi: 10.1002/hep.22317.

[7] Lorenzini S, Isidori A, Catani L, Gramenzi A, Talarico S, Bonifazi F, et al. Stem cell mobilization and collection in patients with liver cirrhosis. Aliment Pharmacol Ther 2008;27:932-939, doi: 10.1111/j.1365-2036.2008.03670.x.

[8] Duan XZ, Liu FF, Tong J], Yang HZ, Chen J, Liu XY, et al. Granulocyte-colony stimulating factor therapy improves survival in patients with hepatitis $B$ virus-associated acute-on-chronic liver failure. World J Gastroenterol 2013; 19:1104-1110. doi: 10.3748/wjg.v19.i7.1104.

[9] Garg V, Garg H, Khan A, Trehanpati N, Kumar A, Sharma BC, et al. Granulocyte colony-stimulating factor mobilizes CD34(+) cells and improves survival of patients with acute-on-chronic liver failure. Gastroenterology 2012;142: 505-512.e1. doi: 10.1053/j.gastro.2011.11.027.

[10] Xing TJ, Xu HT, Xian JC, Shen ML, Li H, Ye J, et al. Mechanism and efficacy of mobilization of granulocyte colony-stimulating factor in the treatment of chronic hepatic failure. Hepatogastroenterology 2013;60:170-175. 


\section{Qiao Y. et al: G-CSF therapy for liver failure}

[11] Singh V, Sharma AK, Narasimhan RL, Bhalla A, Sharma N, Sharma R. Granulocyte colony-stimulating factor in severe alcoholic hepatitis: a randomized pilot study. Am J Gastroenterol 2014;109:1417-1423. doi: 10.1038/ajg. 2014.154.

[12] Rolando N, Clapperton M, Wade J, Wendon J. Administering granulocyte colony-stimulating factor to acute liver failure patients corrects neutrophil defects. Eur J Gastroenterol Hepatol 2000;12:1323-1328. doi: 10.1097/ 00042737-200012120-00010.

[13] Jadad AR, Moore RA, Carroll D, Jenkinson C, Reynolds DJ, Gavaghan DJ, et al. Assessing the quality of reports of randomized clinical trials: is blinding necessary? Control Clin Trials 1996;17:1-12. doi: 10.1016/0197-2456(95) 00134-4.

[14] Schiano TD, Kim-Schluger L, Gondolesi G, Miller CM. Adult living donor liver transplantation: the hepatologist's perspective. Hepatology 2001;33:3-9. doi: 10.1053/jhep.2001.21489.

[15] Piscaglia AC, Arena V, Passalacqua S, Gasbarrini A. A case of granulocytecolony stimulating factor/plasmapheresis-induced activation of granulocytecolony stimulating factor-positive hepatic progenitors in acute-on-chronic liver failure. Hepatology 2015;62:649-652. doi: 10.1002/hep.27708.

[16] Gurakar A, Fagiuoli S, Gavaler JS, Hassanein T, Jabbour N, Wright HI, et al. The use of granulocyte-macrophage colony-stimulating factor to enhance hematologic parameters of patients with cirrhosis and hypersplenism. J Hepatol 1994;21:582-586. doi: 10.1016/S0168-8278(94)80105-3.

[17] Maiwal R, Kumar A, Sarin SK. Liver regeneration during acute-on-chronic liver failure using growth factors: in vivo or ex vivo indulgence of bone marrow? Gastroenterology 2013;145:901-904. doi: 10.1053/j.gastro.2013.08.031.

[18] Lyra AC, Soares MB, da Silva LF, Braga EL, Oliveira SA, Fortes MF, et al. Infusion of autologous bone marrow mononuclear cells through hepatic artery results in a short-term improvement of liver function in patients with chronic liver disease: a pilot randomized controlled study. Eur J Gastroenterol Hepatol 2010;22:33-42. doi: 10.1097/MEG.0b013e32832eb69a.

[19] Salama H, Zekri AR, Medhat E, Al Alim SA, Ahmed OS, Bahnassy AA, et al. Peripheral vein infusion of autologous mesenchymal stem cells in Egyptian
HCV-positive patients with end-stage liver disease. Stem Cell Res Ther 2014; 5:70. doi: 10.1186/scrt459.

[20] Wan Z, You S, Rong Y, Zhu B, Zhang A, Zang H, et al. CD34+ hematopoietic stem cells mobilization, paralleled with multiple cytokines elevated in patients with HBV-related acute-on-chronic liver failure. Dig Dis Sci 2013; 58:448-457. doi: 10.1007/s10620-012-2458-z.

[21] Rolando N, Clapperton M, Wade J, Panetsos G, Mufti G, Williams R. Granulocyte colony-stimulating factor improves function of neutrophils from patients with acute liver failure. Eur J Gastroenterol Hepatol 2000;12:1135-1140. doi: 10.1097/00042737-200012100-00011.

[22] Han Y, Yan L, Han G, Zhou X, Hong L, Yin Z, et al. Controlled trials in hepatitis B virus-related decompensate liver cirrhosis: peripheral blood monocyte transplant versus granulocyte-colony-stimulating factor mobilization therapy. Cytotherapy 2008;10:390-396. doi: 10.1080/14653240802129901.

[23] Yannaki E, Athanasiou E, Xagorari A, Constantinou V, Batsis I, Kaloyannidis P, et al. G-CSF-primed hematopoietic stem cells or G-CSF per se accelerate recovery and improve survival after liver injury, predominantly by promoting endogenous repair programs. Exp Hematol 2005;33:108-119. doi: 10. 1016/j.exphem.2004.09.005.

[24] Theocharis SE, Papadimitriou LJ, Retsou ZP, Margeli AP, Ninos SS, Papadimitriou JD. Granulocyte-colony stimulating factor administration ameliorates liver regeneration in animal model of fulminant hepatic failure and encephalopathy. Dig Dis Sci 2003;48:1797-1803. doi: 10.1023/A:1025463532521.

[25] Piscaglia AC, Shupe TD, Oh SH, Gasbarrini A, Petersen BE. Granulocytecolony stimulating factor promotes liver repair and induces oval cell migration and proliferation in rats. Gastroenterology 2007;133:619-631. doi: 10 . 1053/j.gastro.2007.05.018.

[26] Khanam A, Trehanpati N, Garg V, Kumar C, Garg H, Sharma BC, et al. Altered frequencies of dendritic cells and IFN-gamma-secreting T cells with granulocyte colony-stimulating factor (G-CSF) therapy in acute-on- chronic liver failure. Liver Int 2014;34:505-513. doi: 10.1111/liv.12415. 